\title{
Un momento del azul. Rubén Darío acuña un color
}

\author{
Javier PÉREZ \\ Universidad Complutense de Madrid
}

\begin{abstract}
RESUMEN
Aunque la presencia del color "azul" es de larga tradición, el uso que de él hace Darío lo convierte en el símbolo máximo del Modernismo. En este artículo se hace una revisión de dicho color en la poesía y de los matices que el poeta nicaragüense le otorga.
\end{abstract}

Palabras clave: Darío, colores, azul, modernismo.

\section{A Moment of Blue. Rubén Darío Coins Color}

\begin{abstract}
Although the presence of "blue" has a long tradition, he does use that Darío makes it the ultimate symbol of modernism. This article is a review of that color in the poetry and the nuances that gives Nicaraguan poet.
\end{abstract}

Keywords: Darío, colors, blue, modernism.

Rubén Darío ha sido el poeta que ha conseguido, con una palabra, un movimiento estético. Aunque él no fuese el que acuñase el término de "modernismo", ni tampoco asentase las bases retóricas que se deben más a José Martín , sí consagró el "azul" cuando en español no era aún más que una simpleza diferenciar los colores por atributos simbólicos. El color ha sido una bandera propia de un grupo transatlántico, que fue alzada en México por Manuel Gutiérrez Nájera como título de su revista que

${ }^{1}$ Fue el Papa Pío X al condenar a una secta que bogaba por una religión más subjetiva y personal en su encíclica Pascendi en 1907. Evidentemente el término era peyorativo al principio, pero luego perdió este carácter en cuanto el grupo lo admitió (Véase Ferreiro Villanueva).

${ }^{2}$ Por ejemplo el término del "pájaro azul" lo usa en su primera novela Amistad Funesta, para referirse a jóvenes que buscan pájaros azules en el mercado. El azul será, en la novela, un constante. Desde la primera página del primer capítulo la luz será "azul". Sin embargo, el símbolo no tendrá la fuerza que en el libro de Darío, ya que en Martí también existe la polaridad negro/azul (Véase Martí: 3 y 8). 
difundía el modernismo. La fuente del título de $A z u l . .{ }^{3}$ ha sido inquietante desde su segundo prólogo, la carta de Juan Valera. Este crítico, que sustituyó en su segunda edición al prólogo de Eduardo de la Barra es de una intuición clara aunque especialmente lejana con el movimiento literario que por entonces se fragua. Lo primero que hace el académico es saltar sobre la profundidad de sugerencia del color:

Para mí, tanto vale decir que el arte es lo azul, como decir que es lo verde, lo amarillo, lo rojo. ¿Por qué, en este caso, lo azul [...] ha de ser cifra, símbolo y superior predicamento que abarque lo ideal, lo etéreo, lo infinito, la serenidad el cielo sin nubes, la luz difusa, la amplitud vaga y sin límites, donde nacen, viven, brillan y se mueven los astros? Pero aunque todo esto y más surja del fondo de nuestro ser y aparezca a los ojos del espíritu, evocado por la palabra azul, ¿qué novedad hay en decir que el arte es todo esto? (Valera: 9)

Valera atribuye a Víctor Hugo la idea del título: “l'Art c'est l'azur", que sería el final del siguiente párrafo que le va, ni como anillo al dedo, a las intenciones políticas del libro que publica el nicaragüense:

Quoi! L'art décroîtait pour s'être élargi! Non. Un service de plus, c'est une beauté de plus.

Mais on se récrie. Entreprendre la guérison des plaies sociales, amender les codes, dénoncer la loi au droit, prononcer ces hideux mots, bagne, argousin, galérien, fille publique, contrôler les registres d'inscription de la police, rétrécir les dispensaires, sonder le salaire et le chômage, goûter le pain noir du pauvre, chercher du travail à l'ouvrière, confronter aux oisifs du lorgnon les paresseux du haillon, jeter bas la cloison de l'ignorance, faire ouvrir des écoles, montrer à lire aux petits enfants, attaquer la honte, l'infamie, la faute, le vice, le crime, l'inconscience, prêcher la multiplication des abécédaires, proclamer l'égalité du soleil, améliorer la nutrition des intelligences et des coeurs, donner à boire et à manger, réclamer des solutions pour les problèmes et des souliers pour les pieds nus, ce n'est pas l'affaire de l'azur. L'art, c'est l'azur. ${ }^{4}$

${ }^{3}$ En esta nota no se añade nada nuevo a las génesis del título ya formuladas por Marasso y una larga lista de críticos que se han ocupado del tema con seriedad y profundidad. Si al lector le interesan algunas de las posibilidades más marginales de filiación literaria de 'azul', puede consultar el artículo de José María Martínez.

4 “¡No! El arte no se empequeñece porque se ensanche. En absoluto. Un servicio de más es una belleza de más. // pero todavía hay protestas. Emprender la curación de las plagas sociales, corregir los códigos, denunciar la ley al derecho, pronunciar palabras odiosas, como presidio, polizonte, galeote, prostituta, controlar los registros de inscripción de la policía, limitar los dispensarios, examinar los salarios y el desempleo, probar el pan negro del pobre, buscar trabajo para la obrera, poner frente a frente a los ociosos con monóculo y a los harapientos perezosos, derribar las murallas de la ignorancia, abrir escuelas, enseñar a leer a los niños, combatir la vergüenza, la infamia, la falta, el vicio, el crimen, la inconsciencia, predicar la multiplicación de abecedarios, proclamar la igualdad del sol, mejorar la nutrición de las inteligencias y los corazones, dar de beber y de comer, reclamar soluciones para los 
En este libro es donde Hugo escribe con gran fuerza retórica sobre la unión que en su tiempo se estaba fraguando en torno a la reconstrucción del papel del poeta. En William Shakespeare Victor Hugo une al héroe con el profeta, de alguna manera como lo habían unido ya Byron y William Blake. El producto es que quien aspire a escribir versos tendrá que ser una especie de valedor cultural del futuro. Esta posición de privilegio es muy tentadora y utilizar sólo el color como símbolo de entroncarse con estas dos idealidades (el profeta y el héroe unido en un solo color) es una sugerencia que cautiva. Si bien en la cita de Victor Hugo, "L'Art, c'est l'azur", si el lector permite alguna licencia, se puede traducir como "El arte es el cielo", lo que no desviaría el sentido, sino que reconstruiría la fuerza retórica que tenía en francés. En esta traducción se puede apreciar la metonimia que une 'cielo' y 'azul' y abre la puerta de sugerencia para leer una obra llena de blancos, verdes y, sobre todo, dorados. Rubén Darío pone así el azul frente a una serie de colores. Es la elección de un tiempo, realmente de una portada que se lleva fraguando en la cultura de Europa desde hace unos siglos. Elegirlo es impulsar la cristalización de un cambio.

Para la fuente de $A z u l . .$. Rubén Darío también nos ofrece su versión, negando conocer la cita en prosa de Hugo, prefiere recordar unos versos:

Adieu, patrie,

L'onde est en furie!

Adie, patrie,

Azur! $!^{5}$

Alegando que es un color oceánico, helénico y cita a Ovidio - cita en la que no aparece el azul, sino la luz (Darío, 1988: 38-39)-, aunque es probable que esto sea una reconstrucción cultural de Darío, ya que especialistas aseguran que en las épocas clásicas y de la decadencia el color azul no era muy bien recibido por griegos y romanos $^{6}$. Es un error bastante común donde al contrario de lo que se piensa (como en el caso del Raymond Skyrme) el color azul no es ni de los más antiguos en las corrientes griegas, latinas o hispánicas, y mucho menos uno de los símbolos más comunes. Por ejemplo ni siquiera aparece dentro de la gama alquímica (que es blanco, amarillo, rojo y negro). Quizá esta rareza alentara a Darío y a todo el ambiente parnasiano y modernista. El color azul era, por lo tanto, un color por poblar y eso se acercaba más al futuro que otros colores ya cargados como el blanco o el negro. Pero esta suposición de que el futuro es cercana a Darío ya que su obra es una unión entre el espíritu romántico (provisto con otra sensibilidad) y las condiciones prácticas de las

problemas, y zapatos para los descalzos; todo eso no es asunto del azul. El arte es el azul" (Hugo: 278). / Azul."

La estrofa musical de Les châtiments. “Adiós, patria, / el mar está furioso / Adiós, patria,

6 "Más aún que los griegos, los romanos ven en el azul un color sombrío, oriental o bárbaro; lo empelan muy poco. Para ellos el color de la luz no es el azul, sino el rojo" (Pastoureau: 30). 
contradicciones capitalistas ${ }^{7}$. El "futuro" es uno de los símbolos mejor utilizados en nuestro presente y si no sólo hay que elegir cualquier predicción del FMI o elementos bursátiles que sólo son aproximaciones o recreaciones que rigen un sistema global como si fuesen de una materialidad inmanente.

Ante la búsqueda de su filiación simbólica está bien que Darío quiera escurrirse a la crítica ${ }^{8}$. Lo hizo en vida con su poesía. Sin embargo, lo que probablemente tenía en mente ${ }^{9}$ era la carga cultural vibrante de la época donde el color no sólo era la longitud de onda que existe entre los 460 y los $482 \mathrm{mn}$., o el color casi neutro que hoy predomina en pantalones vaqueros, carpetas y tintas de bolígrafos y rotuladores. El azul contiene un valor metonímico imprescindible: es el cielo y el mar. Es, por lo tanto, la altura y la profundidad, que se convierten en análogas para simbolistas y en resolución de la dualidad día y noche (ambos azules) para los parnasianistas. En francés hay un azul que es "bleu", pero hay otro que es sacro: "azur" el siglo XIX de tumbas egipcias con los techos pintados de azur pudo inculcar en el imaginario social la idea de un azul digno de la muerte del más allá de los dioses vivos que eran los faraones ${ }^{11}$. En Francia fue la insignia monárquica detrás de las flores de lis doradas, y la libertad que sobrevivió en las distintas repúblicas y en las revoluciones. Darío rendía una y otra vez tributo a los poetas franceses, a quienes consideraba sus mentores espirituales y él pudo sentir que escribir "bleu" no es lo mismo que escribir "azur" y mucho menos en poesía.

En Baudelaire el azur es una aspiración continua. Uno de los temas en común con Darío es la inutilidad del poeta en el mundo práctico. Para ambos el poeta es un rey destronado. Baudelaire lo muestra en el verso "ces rois de l'azur" albatros, símbolo de los poetas que aparece en el segundo poema de Flores del mal. Darío se vale del mismo tema en el primer relato del libro, "El rey burgués". Otra de

${ }^{7} \mathrm{Al}$ respecto se puede consultar el inteligente artículo de Goodrich.

${ }^{8}$ Y más si el texto con el que enlazan $A z u l \ldots$ viene de un capítulo que comienza con las frases “ $¡ A$ Ah, genios! ¡Sed útiles! No os hagáis los delicados cuando de lo que se trata es de ser eficaces y buenos. El arte por el arte puede ser hermoso, pero el arte por el progreso aún lo es mucho más". Justo el reverso de la moneda de la actitud contestataria del joven Darío.

${ }^{9}$ Para Darío, en un cuento publicado en Chile, que precede al libro del que nos ocupamos, titulado "Carta del país azul" y que se subtitula "Paisajes de un cerebro", contiene esta definición de las cualidades del azul: "Ayer vagué por el país azul. Canté a una niña; visité a un artista; oré, oré como un creyente en un templo, yo el escéptico; y yo, yo mismo, he visto a un ángel rosado que desde su altar lleno de oro, me saludaba con las alas" (Darío, 1990).

${ }^{10}$ Véase Chevalier y Gheerbrant.

${ }^{11}$ Para José Martí, según Ivan A. Schulman el Azul es el que se vincula "con un mundo espiritual que alcanzan los que se han sacrificado al servicio de la humanidad" (Schulman: 263).

12 "Esos reyes del azul” (Baudelaire: 90). 
las posibles influencias es Verlaine, santo patrón de los modernistas y bohemios ${ }^{13}$, en uno de sus poemas dedicados a su persona, habla de sí y de sus ambiciones y utiliza el azur: "La fenètre est si petite là-haut qu'elle ne permet de voir que l'azur."14. Como vemos los azules poéticos que parecen rodear a $A z u l .$. son de una intención que van a la par del trabajo de Rubén Darío. Barrionuevo sugiere que fue un libro casi improvisado, animado por la concesión de un premio literario. Si esto es verdad demuestra la precisión de intuición de términos culturales que Darío maneja, no así su hondura de recopilación bibliográfica, que, paradójicamente, hacen hueras estas explicaciones. Pero su poesía nos deja la comprensión de un tiempo. A finales del siglo XIX el azul era un color tópico de las poesías elitistas francesas. Mallarmé y su poema "L'Azur" pueden servir como ejemplo ${ }^{15}$.

Kandinsky, casi de la misma promoción que Darío, ya que sólo son cinco semanas uno mayor que otro (un poquito más de lo que los distancia los dos calendarios en que nacieron) basa el azul en la puerta a la dimensión interior. Ambos, que pertenecen a la "periferia" del arte eurocentrista del momento, son pilares sobre los que se construye en el siglo XX. El salto de pértiga en pintura de Kandinsky, es análogo al trabajo de Darío en lengua castellana. Para Kandinsky "el azul es el color típicamente celeste que desarrolla profundamente el elemento de quietud" es la fuerza profundizadora que penetra en el alma de forma directa con cualquier forma geométrica.

La tendencia del azul hacia la profundidad es tan grande que precisamente en los tonos profundos adquiere mayor intensidad y fuerza interior. [...] La llamada infinita que despierta en él su deseo de pureza e inmaterialidad. (Kandinsky: 74)

Estas ideas son tributarias de la posición que Goethe confiere al color azul, aunque el pintor no se limita a parafrasear, añade dos atributos al color: la flauta y el círculo (Kandinsky: 58).

En su Teoría de los colores, Goethe pone al azul en un extremo. Es el extremo interior del mundo, hacia el que siempre nos acercamos pero es imposible llegar, es singular e inefable. Este color es una energía y en su lado más puro es "una preciosa nada", donde se mezclan excitación y serenidad (Goethe: 206-207).

${ }^{13}$ Sólo con recordar la última visión de Max Estrella, personaje de Luces de Bohemia, antes de morir da una idea. Se le presenta un funeral, que es el de Verlaine, como si quisiera ver ahí el suyo propio.

14 "Le Faible Verlaine": “Allá arriba la ventana es tan pequeña que no deja ver otra cosa que el cielo" (Verlaine: 248).

${ }^{15}$ El poema acaba exclamando "l'Azur triomphe, et je l'entends qui chante / Dans les cloches. Mon âme, il se fait voix pour plus / Nous faire peur avec sa victoire méchante, / Et du metal vivant sorte en bleus angélus! // [...] Oú fuir dans le révolte inútil et perverse? / Je suis hanté. L’Azur! L’Azur! 1Azur! L’Azur!” que traducido podría escribirse así: “¡El Azur triunfa, alma mí, lo escucho / cantando en las campanas convirtiéndose en voz / para atemorizarnos con su triunfo maligno, / del metal vivo sale en ángelus azules! // [...] ¿A dónde huir en la inútil revuelta perversa? / Soy un poseso ¡Azur! ¡Azur! ¡Azur! ¡Azur!” (Mallarmé: 60). 
Este es el momento más alto del color azul donde, después de ser considerado un color puro, se lo toma como polo del eje amarillo-azul que predominará en todos aquellos que hubiesen leído a Goethe y lo prefieran a Newton. Y esto es extraño debido a que parece que hasta las vocaciones marianas del siglo XII no existe el color azul, como, hasta hace bien poco, no existía de forma clara el color azul en Japón ${ }^{16} \mathrm{o}$ en la lengua mbembe ${ }^{17}$. Este color no aparecía en los tratados de pintura de Plinio el Viejo ${ }^{18}$ y de otros espacios de alta cultura (como colores admitidos o rechazados por la liturgia cristiana durante el primer milenio). Hasta el XIII no se impone como color oficial del manto, el vestido o algún atributo de la virgen María y a la Inmaculada Concepción. Desde ese momento, tiene una ascensión fulgurante ${ }^{19}$.

¿Qué sería de la pintura sin el azul para el Bosco? ¿Y el Greco? ¿Y Vermeer? Las obras de ascensión, de interiorización, de luz opacada gana, con estos autores un lugar de ensalzamiento, humano, pero a la vez divino. El azul crece con Europa, la que ya no es de Roma y Grecia. Los reyes van sumando ese color a las heráldicas. Y en 1709 se inventa el azul de Prusia, lo que impulsará a este color que era costoso debido al comercio del valioso índigo y la relativa pobreza material de sus alternativas.

Goethe es el que, con su Werther, impone el color azul como el compromiso del amor y la moda juvenil:

Me costó mucho decidirme a abandonar el sencillo frac azul que llevaba cuando bailé por primera vez con Carlota; pero al final estaba demasiado raído. Encargué otro exactamente igual, con un chaleco y unas calzas amarillas idénticas a las que llevaba aquel día. (Carta del 6 de septiembre de 1772 de Werther a Wilhem)

El color azul ha pasado así de ser un color de la fuerza natural que recibe lo divino (encarnado por la Virgen María) a un uso que va expresándose con más naturalidad y menos divinidad, según la sociedad también va secularizando sus expresiones. Es, como he escrito, el color más joven. Sin connotaciones alquímicas o representaciones artísticas de la antigüedad más allá de las tumbas egipcias y algunos restos micénicos que costará descubrir. El azul en Darío es un símbolo del juego doble de la cultura, siempre inexacta, pero con la sensación de permanencia inamovible que animaba el influjo positivista en la poesía (la autarquía que podía hacer resaltar a las artes si se unían y se intercambiaban). Aunque el positivismo parezca de carácter materialista,

${ }^{16}$ En japonés las palabras 'midori' y'ao', significan hoy 'verde' y 'azul', aunque antes se utilizaban indistintamente. Hoy en día a la luz verde de los semáforos se la sigue llamando 'ao'.

${ }^{17}$ La lengua mbembe, de Nigeria, usa el término 'obina' para 'azul', 'verde' y 'negro'.

18 "Fue utilizando únicamente cuatro colores cómo Apeles, Aethion, Melantio y Nicómaco, los pintores más célebres, ejecutaron las inmortales obras de arte que todos conocen. Para los blancos, el melinum; para los amarillos, el sil ático; para los rojos, la sinopis del Ponto; para los negros, el atramentum" (párrafo XXXII).

${ }^{19}$ Véase Pastoureau. 
no es para nada inmanente ${ }^{20}$. En manos de Darío el término ha sufrido una breve revitalización numinosa a través de esta grieta de revelación que abren los poetas franceses (recordemos que "azur" es un término sagrado), pero esto ha extrañado el color de la monarquía, quizá convirtiéndolo en igualitario de libertad y sublimación. Será entonces cuando comience a utilizarse como una forma de rareza parecida al giro del "perro verde" que existe en español. El 'azul' acompaña a un mito francés del siglo XIX que se extiende como personaje de cuento en el que un pájaro azul es el pájaro de la felicidad. Este es el que motiva el cuento de Darío donde un escritor se enajena (debido a la mitificación) y que Maeterlink versionará, no directamente a Darío, pero sí con curiosas similitudes, en 1908, en otro "Pájaro Azul de la felicidad" ${ }^{21}$. En Maeterlinck el azul pasa de ser revelación a ser sueño ${ }^{22}$.

Para terminar me gustaría quedarme con la imaginación de Juan Ramón Jiménez que fue "llamado" por Darío para luchar en las filas de la poesía cuando contaba con dieciocho años. Su primer viaje a Madrid es por la carta del nicaragüense al que, en ese momento, consideraba el "Napoleón" de la poesía. En su memoria madura Juan Ramón Jiménez ve al poeta vestido de blanco (quizá de lino, quizá de luz), gravitando entre inmensidades abiertas "Atesorador de su designio, libre ya de aquel "destierro" de periodista del mar, que era su melancolía, [...] sin otra utilidad que su belleza parnasiana, serán lujo de su casa flotante entre dos espacios, aire y agua. ¡El azul, el doble azul!" (Jiménez: 45).

Darío se ató así a un color que representaba, probablemente sin él saberlo, la apuesta estética de Occidente independiente de su pasado y lejos de las convenciones culturales de otros países. Si hay, por lo tanto, propuestas culturales propias en Europa están en el color azul, del que, paradójicamente, un poeta nicaragüense nos hizo resaltarlo en español. Era, en realidad, una más de las múltiples vías por las que la poesía entroncó con su presente y su futuro.

\section{BIBLIOGRAFÍA}

BAUDELAIRE, Charles.

1998 Las flores del mal. Madrid: Cátedra.

CIRLOT, Juan-Eduardo.

1982 Diccionario de los símbolos. Barcelona: Labor.

CHEVALIER, Jean y GHEERBRANT, Alain.

2007 Diccionario de los símbolos. Barcelona: Herder.

${ }^{20}$ Por ejemplo, Auguste Comte creía en un futuro revelado y pretendía fundar su unidad en una religión donde la divinidad suprema fuese la "Humanidad".

21 George Cukor rueda una película sobre este libro como guión en 1976 (Véase Maeterlink).

${ }^{22}$ Véase Sánchez Sánchez. 
DARÍO, Rubén.

1984 Azul... Madrid: Espasa-Calpe.

1988 Historia de mis libros. Managua: Nueva Nicaragua.

1990 Cuentos completos. La Habana: Arte y literatura.

FERREIRO VILLANUEVA, Cristina.

1990 La obra poética de Rubén Darío. Madrid: Ciclo.

Goethe, J.W.V.

1999 Teoría de los colores. Madrid: Celeste.

GOODRICH, Diana Sorensen.

1985 "Azul...: Los contextos de lectura", Hispamérica. Revista de Literatura, vol. 14, $\mathrm{n}^{\circ} 40$, Hartwick.

HugO, Victor.

1880 William Shakespeare. Paris: Nelson.

JIMÉNEZ, Juan Ramón.

1990 Mi Rubén Dario. Moguer: Fundación Juan Ramón Jiménez.

KANDINSKY, Vasili.

2004 De lo espiritual en el arte. Barcelona: Paidós.

MAETERLINK, Maurice.

2000 La intrusa. Los ciegos. Pelléas y Melisande. El pájaro azul. Madrid: Cátedra.

MARTÍ, José.

2000 Amistad Funesta. Buenos Aires: Ediciones El Aleph.

MARTíNEZ, José María.

1996 "Nuevas luces para las fuentes de Azul...", Hispanic Review, vol. 64, $\mathrm{n}^{\mathrm{o}} 2$, Filadelfia, University of Pennsylvania Press.

MALLARMÉ, Stéphane.

1980 Obra poética. Vol. 1. Madrid: Hiperión.

MATAMORO, Blas.

2002 Rubén Dario. Madrid: Espasa.

PASTOUREAU, Michel.

2010 Azul... Historia de un color. Barcelona: Paidós.

PLINIO EL VIEJO.

1999 Historia natural. Vol. V. México: Visor.

RUIZ BARRIONUEVO, Carmen.

2002 Rubén Darío. Madrid: Síntesis.

SÁNCHEZ SÁNCHEZ, Juan Pedro.

2003 “Ética y estética en El pájaro azul de Maeterlinck”, Espéculo, n 24 (julio-octubre)

(http://www.ucm.es/info/especulo/numero24/p_azul.html) [16-052011].

SCHULMAN, Iván A.

1960 "Génesis del azul modernista", Revista iberoamericana, vol. 25, Pittsburgh, University of Pittsburgh. 
SKYRME, Raymor.

1968 "Dario's Azul...: A Note on the Derivation of the Title", Romance Notes, vol.10, Chap Hill, Carolina del Norte.

VERLAINE, Paul.

1981 Poesía completa. Tomo I. Madrid: Río Nuevo. 